\title{
Isolated Spontaneous Primary Tubercular Erector Spinae Abscess: A Case Report and Review of Literature
}

\author{
Bhavuk Garg, Chaitanya Dev Pannu, Rishi Ram Poudel, Vivek Morey \\ Department of Orthopedics, All India Institute of Medical Sciences, New Delhi, India
}

Isolated spontaneous primary tubercular erector spinae abscess in an immunocompetent patient is very rare. Here, we report such a case of 21-year-old female, which was successfully managed with timely diagnosis and intervention. Isolated primary tubercular abscess of erector spinae is a rare differential diagnosis of low back pain; however, it must be suspected in an endemic region for tuberculosis, especially when raised erythrocytic sedimentation rate and C-reactive protein are present. Excision along with anti tubercular therapy proved to be a successful strategy in our patient.

Keywords: Erythrocytic sedimentation rate; C-reactive protein

\section{Introduction}

Isolated primary involvement of the skeletal muscles by Mycobacterium tuberculosis is very rare, probably because skeletal muscles does not provide an adequate medium for the growth of this bacteria. Erector spinae is deep skeletal muscle of back, and several reports $[1,2]$ have described primary pyogenic erector spinae abscess along with extradural abscess. However, there has been only one report [3] of isolated spontaneous primary tubercular erector spinae abscess to date. The significance of this case report lies in the very fact that while evaluating a case of lower back pain, a physician should also consider isolated spontaneous tubercular abscesses in the differential diagnosis, particularly in endemic region where the incidence of musculoskeletal tuberculosis is very high.

\section{Case Report}

A 21-year-old patient presented to us with the complaints of insidious onset lower back pain for the previous 2 months. The pain was mild in intensity to begin with and progressive in nature, non-radiating, and of a generalized dull aching nature, with no diurnal variations. It was associated with malaise but no fever. She was initially treated conservatively for 2 weeks with non-steroidal anti inflammatory drugs (NSAIDs) but was not relieved. The pain's intensity continued to increase, and she started having mild grade fever, particularly in the evenings. She had no recent history of corticosteroid use or chemotherapy, direct contact with a person having pulmonary tuberculosis, or history of any significant medical illness or an immunocompromised state. On examination, the bilateral straight leg raise test was negative with paravertebral muscle spasms and tenderness mainly located in

Received Mar 6, 2014; Revised Oct 1, 2014; Accepted Oct 16, 2014

Corresponding author: Chaitanya Dev Pannu

Department of Orthopedics, All India Institute of Medical Sciences, New Delhi [110029], India

Tel: +911126593550, Fax: +919968037279, E-mail: cdpannu@gmail.com 
the right lumbar region without any focal neurological deficit.

Laboratory results showed leucocytes within normal range $(9,100$ cells/cumm), lymphocytosis $(36 \%)$, an elevated erythrocyte sedimentation rate $(80 \mathrm{~mm} / \mathrm{hr}$; normal range, $0-20 \mathrm{~mm} / \mathrm{hr})$ and C-reactive protein level $(22 \mathrm{mg} /$ $\mathrm{L}$; normal range, 0-6 $\mathrm{mg} / \mathrm{L}$ but may vary from lab to lab). All other lab parameters, such as blood sugar, renal function tests and liver function tests were normal. Chest and lumbar spine radiographs were normal except for the loss of lumbar lordosis in the lumbar spine. Magnetic resonance imaging (MRI) showed a well-defined cystic lesion in right sided erector spinae muscle at the level of L5 vertebra with perilesional edema, which was hyperintense in T1-weighted images and hypointense in T2-weighted images, highly suggestive of an abscess (Figs. 1,2). There was no area of abnormal signal intensity in the adjacent spine. A differential diagnosis of pyogenic abscess, tuber-

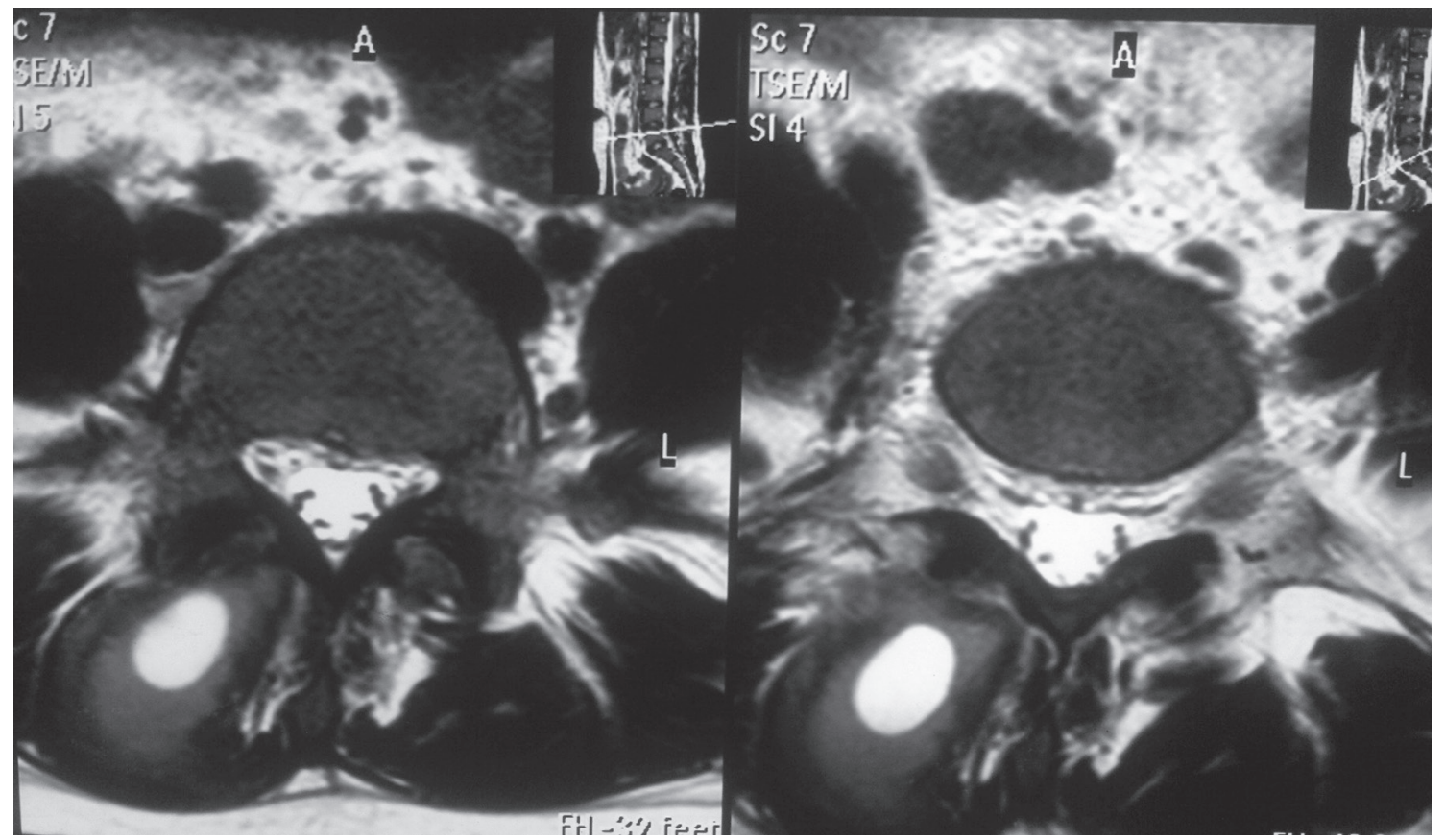

Fig. 1. Magnetic resonance imaging of patient.

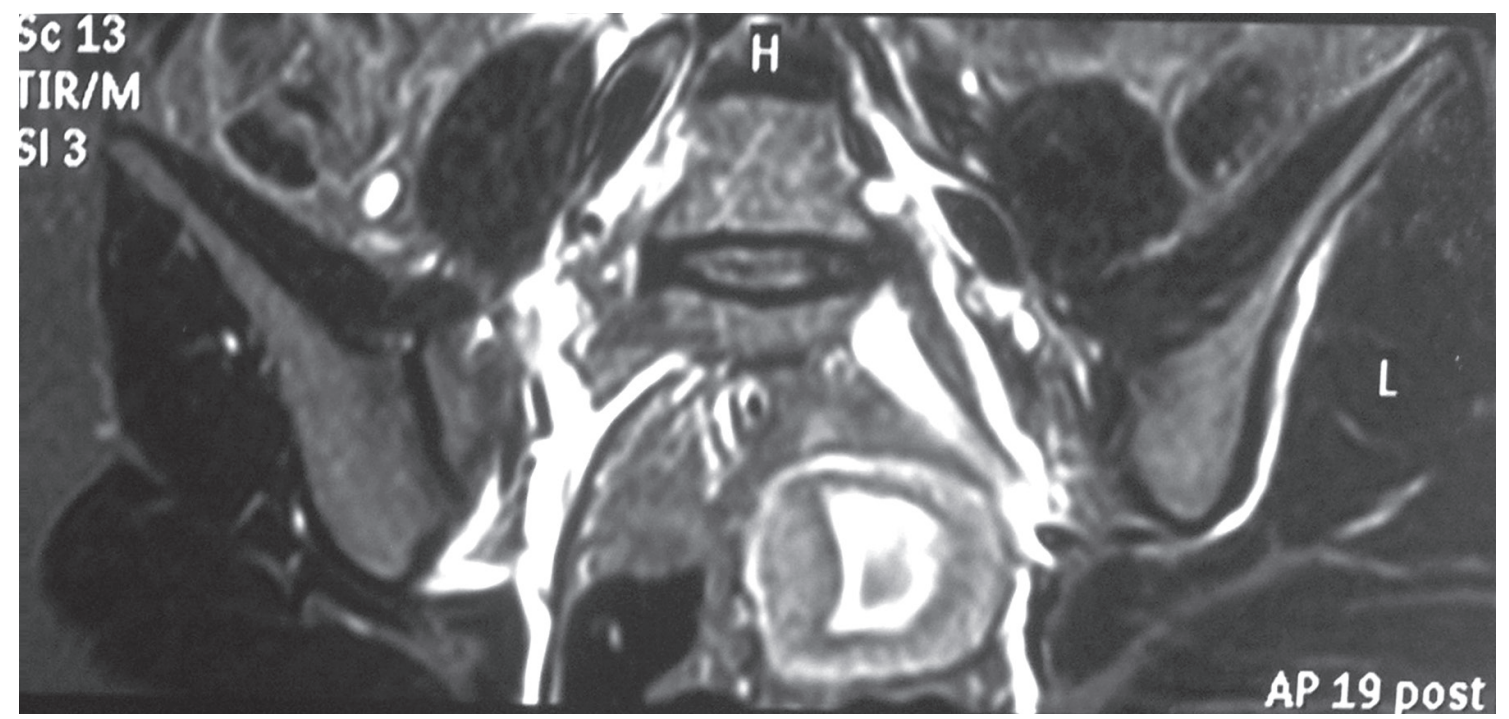

Fig. 2. Magnetic resonance imaging of patient 
Table 1. Previous case reports of primary muscular tuberculous abscesses

\begin{tabular}{|c|c|c|c|}
\hline Serial no. & Study (author, journal, year) & Muscle involved & Immunity status \\
\hline 1. & Ahmed and Homans [4], Pediatr Infect Dis J, 2002 & Soleus & Immunocompromised \\
\hline 2. & Johnson and Herzig [5], Nephrol Dial Transplant, 2000 & Quadriceps & Immunocompromised \\
\hline 3 & Abdelwahab and Kenan [6], J Bone Joint Surg Am, 1998 & Brachialis and biceps brachii & Immunocompetent \\
\hline 4 & Simon et al. [7], Med Ultrason, 2011 & Quadriceps & Immunocompromised \\
\hline 5 & Harrigan et al. [8], J Emerg Med, 1995 & Psoas major & Immunocompetent \\
\hline 6 & Chen [9], Clin Rheumatol, 1998 & Fascia lata & Immunocompetent \\
\hline 7 & Toda et al. [10], Comput Med Imaging Graph, 1998 & Gluteus maximus & Immunocompetent \\
\hline 8 & Varshney et al. [11], Joint Bone Spine, 2007 & Achilles tendon & Immunocompetent \\
\hline 9 & Narang [12], Hand (N Y), 2009 & Biceps brachii & Immunocompetent \\
\hline 10 & Sridhar and Seith [13], Eur J Radiol Extra, 2004 & Temporalis muscle & Immunocompetent \\
\hline 11 & Bhatty et al. [14], JK Science, 2011. & Vastus lateralis & Immunocompetent \\
\hline 12 & Ramakant et al. [15], Indian J Tuberc, 2004 & Rectus abdominis & Immunocompetent \\
\hline 13 & Griffiths [16], J Bone Joint Surg Br, 1956 & Rectus femoris & Immunocompetent \\
\hline 14 & Plummer et al. [17], J Bone Joint Surg Am, 1934 & Gastrocnemius muscle & Immunocompetent \\
\hline 15 & Sokucu et al. [18], J Pak Med Assoc , 2013 & Biceps brachi & Immunocompetent \\
\hline 16 & Mascarenhas et al. [19], Br J Oral Maxillofac, 2009 & Left masseter & Immunocompetent \\
\hline 17 & Trikha and Gupta [20], J Infect, 2002 & Biceps brachii & Immunocompetent \\
\hline 18 & Perez-Alonso et al. [21], Med Mal Infect, 2011 & Right rectus femoris & Immunocompetent \\
\hline 19 & Elshafie et al. [3], Sultan Qaboos Univ Med J, 2013 & Errector spinae & $\begin{array}{l}\text { Immunocompetent with } \\
\text { history of direct contact }\end{array}$ \\
\hline
\end{tabular}

cular abscess, and cysticercosis was made. Cysticercosis was ruled out by ultrasound. Our suspicion of a pyogenic abscess was less due to the absence of local and systemic signs. A decision to perform a biopsy was made.

A paramedian incision was made and about 1 inch below the skin during the separation of muscle fibers, 2-5 $\mathrm{mL}$ of caseous material was drained, which was sent to the lab for culture sensitivity and acid fast bacillus (AFB) staining. An excisional biopsy was performed and the adjacent soft tissue was sent for histopathology. Puss culture sensitivity for bacteria came out to be sterile and AFB staining was negative. The histopathology report revealed a fibrocollagneous tissue infiltrated by chronic infiltrate cells. A focal giant cell reaction was seen and an ill-defined granuloma was also seen. Hence, the histopathological features were highly suggestive of tuberculosis. The patient was put on anti-tubercular therapy for a total of 9 months and was completely relieved of her symptoms at the final follow-up. A repeat MRI was performed after completion of the anti-tubercular therapy, which showed complete resolution of the disease.

\section{Discussion}

Isolated involvement of the muscles without skeletal or extraskeletal tuberculosis is believed to be a rare occurrence, although few cases have been reported earlier (Table 1) [4-21]. Culotta [22] reported only four cases of the muscle tuberculosis from autopsies of 2,224 tuberculosis patients. Involvement of skeletal muscles is very rare, even in patients with widespread involvement of the disease. Petter [23] recorded only one case of primary muscular tuberculosis in over 6,000 cases of all types of tuberculosis, with a frequency of $0.015 \%$. Mycobacterium tuberculosis spreads very rarely to the muscles through hematogenous or contiguous methods, hence clinical symptoms are very vague, leading to delay in diagnosis and treatment of the disease. Therefore, the muscular involvement is commonly associated with involvement of the adjacent bones and joint. Hematogenous dissemination plays an important role in tuberculars myositis [24]. In one study, half of the patients with hematogenous spread also had involvement of several muscles [25]. Transmission by the 
way of injection has also been reported, in addition to contiguous and hematogenous spread [26]. Manifestations of tubercular myositis may mimic malignant or other inflammatory diseases, leading to misdiagnosis [24]. The most common site of involvement, by far, is the thigh [27]. So far, many muscles have been reported to be affected by isolated primary tuberculosis, including gastronemius, biceps brachii, and psoas major. Isolated abscess of the erector spinae muscle is also very rare. Few cases have been reported as pyomyositis of erector spinae with epidural abscess $[1,2,28]$. Because clinical symptoms are initially very vague and overlap with the usual causes of the back pain, such as in prolapsed intervertebral discs, the exact diagnosis is usually delayed until fever and other constitutional features become obvious. Differentiating pyogenic and tubercular abscesses clinically can be very difficult. The presence of a severe low backache, exquisite tenderness in the paraspinal region, and a high grade fever with rigor and chills favors pyogenic abscesses, whereas insidious onset low back pain, tenderness in the paraspinal region with a low grade on-and-off fever without rigor and chills is more likely to be indicative of a tubercular abscess. In our case, the diagnosis was delayed for 3 weeks. An MRI scan is of the utmost importance in making a diagnosis, as the abscess becomes quite obvious in the MRI scan, showing a hypointense lesion in T1weighted images and hyperintense lesion with typical rim sign in T2-weighted images.

The diagnosis of isolated primary tubercular abscess in erector spinae is very rare and a requires high degree of suspicion, particularly in endemic areas [27]. In the Indian subcontinent and other developing countries where the incidence of musculoskeletal tuberculosis is relatively high, isolated primary tubercular abscesses in the erector spinae should be considered as a differential in cases presenting with lower back pain, especially with constitutional symptoms and in the presence of high erythrocytic sedimentation rate and C-reactive protein. MRI is the imaging modality of choice for diagnosing the soft tissue masses associated with the muscular tuberculosis and histopathology is the gold standard for diagnosis. If there is frank puss, it should be drained and the appropriate anti-tubercular therapy should be started for complete resolution as medical treatment is treatment of choice, although operative intervention may be used as an adjunct.

\section{Conflict of Interest}

No potential conflict of interest relevant to this article was reported.

\section{References}

1. Marshman LA, Bhatia CK, Krishna M, Friesem T. Primary erector spinae pyomyositis causing an epidural abscess: case report and literature review. Spine J 2008;8:548-51.

2. Hassan FO, Shannak A. Primary pyomyositis of the paraspinal muscles: a case report and literature review. Eur Spine J 2008;17 Suppl 2:S239-42.

3. Elshafie KT, Al-Hinai MM, Al-Habsi HA, Al-Hattali MS, Hassan O, Al-Sukaiti R. A massive tuberculosis abscess at the erector spinae muscles and subcutaneous tissues in a young man. Sultan Qaboos Univ Med J 2013;13:601-5.

4. Ahmed J, Homans J. Tuberculosis pyomyosits of the soleus muscle in a fifteen-year-old boy. Pediatr Infect Dis J 2002;21:1169-71.

5. Johnson DW, Herzig KA. Isolated tuberculous pyomyositis in a renal transplant patient. Nephrol Dial Transplant 2000;15:743.

6. Abdelwahab IF, Kenan S. Tuberculous abscess of the brachialis and biceps brachii muscles without osseous involvement. A case report. J Bone Joint Surg Am 1998;80:1521-4.

7. Simon SP, Fodor D, Valasciuc R, Tamas MM, Rednic S. A rare case of primary tuberculous pyomyositis. Case report. Med Ultrason 2011;13:245-8.

8. Harrigan RA, Kauffman FH, Love MB. Tuberculous psoas abscess. J Emerg Med 1995;13:493-8.

9. Chen WS. Tuberculosis of the fascia lata. Clin Rheumatol 1998;17:77-8.

10. Toda K, Yasunaga Y, Takemoto S, Terada Y. MR image and CT scan of a tuberculous abscess in the gluteus maximus muscle. Comput Med Imaging Graph 1998;22:425-7.

11. Varshney MK, Trikha V, Gupta V. Isolated tuberculosis of Achilles tendon. Joint Bone Spine 2007;74:1036.

12. Narang S. Tuberculous pyomyositis of forearm muscles. Hand (N Y) 2009;4:88-91.

13. Sridhar C, Seith A. Tuberculous pyomyositis of the temporalis muscle. Eur J Radiol Extra 2004;52:89-91. 
14. Bhatty SM, Prakash JS, John, B. Primary tuberculous abscess of vastus lateralis muscle. JK Science 2011;13:37-8.

15. Ramakant D, Kalpana D, Hetal S, Keyur S. Tuberculosis abscess of rectus abdominis muscle. Indian J Tuberc 2004;51:231-3.

16. Griffiths E. Tuberculoma of striated muscle. J Bone Joint Surg Br 1956;38:855-61.

17. Plummer WW, Sanes S, Smith WS. Hematogenous tuberculosis of skeletal muscle. J Bone Joint Surg Am 1934;16:631-9.

18. Sokucu S, Sokucu SN, Kabukcuoglu Y, Kabukcuoglu F. Primary skeletal muscle tuberculosis at an unusual site. J Pak Med Assoc 2013;63:126-8.

19. Mascarenhas S, Tuffin JR, Hassan I. Tuberculous submasseteric abscess: case report. Br J Oral Maxillofac Surg 2009;47:566-8.

20. Trikha V, Gupta V. Isolated tuberculous abscess in biceps brachii muscle of a young male. J Infect 2002;44:265-6.

21. Perez-Alonso AJ, Husein-Elahmed H, Duran CP,
Caballero-Marcos L, Ramon JA. Isolated muscle tuberculosis. Med Mal Infect 2011;41:559-60.

22. Culotta A. La-tuberculosis muscuolare. Rev Pathol Tuberc 1929;3:1-26.

23. Petter CK. Some thoughts on tuberculosis of fascia and muscle. Lancet 1937;57:156-9.

24. Plummer WW, Sanes S, Smith WS. Hematogenous tuberculosis of skeletal muscle. J Bone Joint Surg 1934;16:631-9.

25. FitzGerald R, Hutchinson CE. Tuberculosis of the ribs: computed tomographic findings. Br J Radiol 1992;65:822-4.

26. Puttick MP, Stein HB, Chan RM, Elwood RK, How AR, Reid GD. Soft tissue tuberculosis: a series of 11 cases. J Rheumatol 1995;22:1321-5.

27. Wang JY, Lee LN, Hsueh PR, et al. Tuberculous myositis: a rare but existing clinical entity. Rheumatology (Oxford) 2003;42:836-40.

28. Srinivasan US, Jagadeesh PC, Senthil Kumar G. Pyogenic epidural abscess with primary erector spinae pyomyositis-case report. Open Spine J 2010;2:17-20. 